\title{
Vibration of open cylindrical shells: A three-dimensional elasticity approach
}

\author{
C. W. Limª) \\ Department of Civil Engineering, The University of Queensland, Brisbane, Queensland 4072, Australia \\ K. M. Liew \\ Division of Engineering Mechanics, School of Mechanical and Production Engineering, \\ Nanyang Technological University, Singapore 639798 \\ S. Kitipornchai \\ Department of Civil Engineering, The University of Queensland, Brisbane, Queensland 4072, Australia
}

(Received 14 October 1997; revised 8 May 1998; accepted 20 May 1998)

\begin{abstract}
The three-dimensional elastic analysis of the vibration of open cylindrical shells are presented. Transverse normal stress usually neglected in plate and shell higher-order theories has been considered. The natural frequencies and vibration mode shapes have been obtained via a three-dimensional displacement-based extremum energy principle. Excessive requirements for memory and computational effort have been overcome, without sacrificing numerical accuracy, by (i) decoupling the three-dimensional displacements into the product of a set of beam and shell shape functions; and (ii) classifying the vibration modes. The effects of subtended angle and aspect ratio have been concluded for shells with various boundary conditions. Typical vibration mode shapes demonstrating the dependence of vibration characteristics on boundary constraints are presented. (C) 1998 Acoustical Society of America. [S0001-4966(98)01609-9]
\end{abstract}

PACS numbers: 43.40.Ey [CBB]

\section{INTRODUCTION}

Despite the practical importance of elastic vibration solutions to engineering design of thick structures, particularly in armed vehicles and nuclear power plants, direct threedimensional elasticity theory has rarely been exploited in numerical vibration analysis. This is because threedimensional numerical analysis of thick plates and shells requires huge computational memory and long execution hours.

The vibration of thick plates and shells has conventionally been solved using the first-order ${ }^{1,2}$ and higher-order theories. ${ }^{3}$ Solutions to the vibration of thick shallow shells have been presented by Lim and Liew ${ }^{4,5}$ and Liew and $\mathrm{Lim}^{6}$ for singly curved and doubly curved shells with arbitrary boundary conditions. Three-dimensional elastic solutions are particularly scarce and almost all investigations have been concerned with rods and beams, ${ }^{7-9}$ parallelepipeds, ${ }^{10-16}$ cylinders, ${ }^{17-20}$ and hollow cones. ${ }^{21}$ To the authors' knowledge, only closed shells or hollow cylinders ${ }^{18-20}$ and cones ${ }^{21}$ have been investigated. Numerical studies for thick, open cylindrical shells have received relatively little attention despite their common applications in the armament industry and nuclear storage designs such as protective tank walls and thick cylindrical covers.

The closed shells, being bodies of revolution, permit one to assume whole periodic wave numbers $(\sin n \theta$ and $\cos n \theta$ ) in representing displacement variations in the circumferential direction, yielding the proper periodicity in $\theta$. This also permits one to separate out the modes by respective circumferential wave numbers $(n)$, reducing the mathematical com-

\footnotetext{
${ }^{a)}$ Present address: Department of Mechanical Engineering, The University of Hong Kong, Pokfulam Road, Hong Kong.
}

plexity to a set of two-dimensional analyses. For open shells, the assumption of whole periodic wave numbers in the circumferential direction is inappropriate and a set of complete three-dimensional analysis is required. This forms a major deterrent so that analyses of open shells have not been widely available.

In view of the lack of analytical solutions, this paper presents an endeavor to investigate the free vibration characteristics of thick and open shells using a three-dimensional displacement-based extremum energy principle. The strain energy integral considers transverse normal stress which is usually neglected in first-order and higher-order theories. The solutions are therefore exact so far as the energy expression is concerned. A Ritz energy functional is defined and minimized to derive a governing eigenvalue equation. The three-dimensional displacement field is characterized by a cylindrical coordinate system with orthogonal displacement components. Although the analysis is completely threedimensional, excessive requirements for memory and computational effort have been overcome, without sacrificing numerical accuracy, by (i) decoupling the three-dimensional displacements into the product of a set of $p$-Ritz beam and shell shape functions, and (ii) classifying the vibration modes into various symmetry classes. One- and two-dimensional (1-D and 2-D) $p$-Ritz functions are formulated to describe the thickness deformation and the midsurface deformation, respectively. By classifying the vibration modes, memory requirements and execution time can be tremendously reduced while maintaining the same level of numerical accuracy. The effects of subtended angle and aspect ratio have been investigated for shells with various boundary conditions. Typical vibration mode shapes demonstrating the de- 


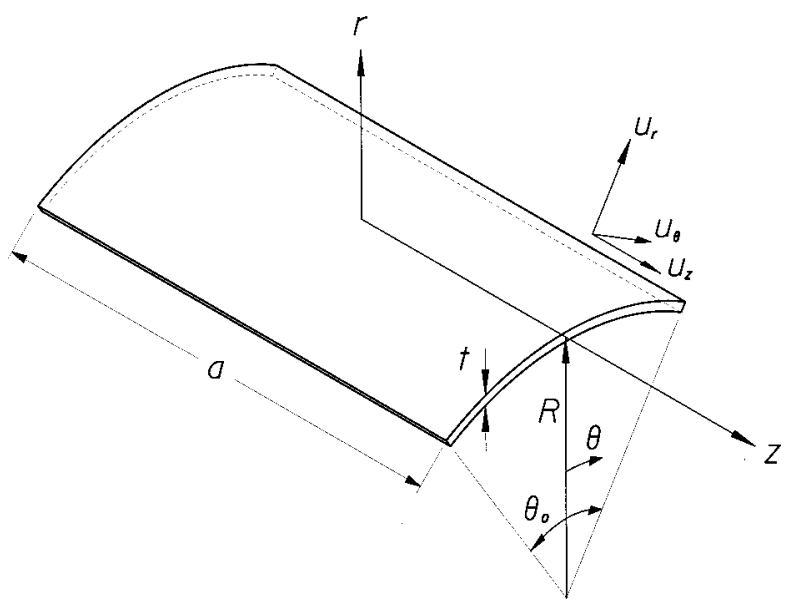

FIG. 1. Geometry of a thick cylindrical shell.

pendence of vibration characteristics on boundary constraints have been presented.

\section{THEORY AND FORMULATION}

\section{A. Basic definition and cylindrical coordinate system}

Consider an isotropic, open and thick cylindrical shell with length $a$, midsurface radius $R$, subtended angle $\theta_{0}$, thickness $t$ as shown in Fig. 1. The circumferential arc length is $b=R \theta_{0}$. An orthogonal cylindrical coordinate system $(r, \theta, z)$ is defined with $r$ the radial coordinate, $\theta$ the angular coordinate, and $z$ parallel to the axis of cylindrical shell. For brevity and generality, a dimensionless coordinate system,

$$
\begin{aligned}
& \bar{r}=\frac{r}{R}, \\
& \bar{\theta}=\frac{\theta}{\theta_{0}}, \\
& \bar{z}=\frac{z}{a},
\end{aligned}
$$

is defined such that the shell is bounded by $1-t / 2 R \leqslant \bar{r} \leqslant 1$ $+t / 2 R,-0.5 \leqslant \bar{\theta} \leqslant 0.5$, and $-0.5 \leqslant \bar{z} \leqslant 0.5$. The midsurface is defined as $\bar{r}=1$. The orthogonal displacement components are $u_{r}, u_{\theta}$, and $u_{z}$.

\section{B. Three-dimensional strain and kinetic energy expressions}

For linear and elastic free vibration, the strain energy of a three-dimensional solid is

$$
\begin{aligned}
U= & \frac{1}{2} \iiint_{V}\left[(\Delta+2 G)\left(\epsilon_{r r}^{2}+\epsilon_{\theta \theta}^{2}+\epsilon_{z z}^{2}\right)\right. \\
& +2 \Delta\left(\epsilon_{r r} \epsilon_{\theta \theta}+\epsilon_{\theta \theta} \epsilon_{z z}+\epsilon_{z z} \epsilon_{r r}\right) \\
& \left.+G\left(\gamma_{\theta z}^{2}+\gamma_{z r}^{2}+\gamma_{r \theta}^{2}\right)\right] r d r d \theta d z,
\end{aligned}
$$

where $V$ is the volume, $G$ is the shear modulus, and

$$
\begin{aligned}
& \Delta=\frac{\nu E}{(1+\nu)(1-2 \nu)}, \\
& G=\frac{E}{2(1+\nu)},
\end{aligned}
$$

in which $E$ is the Young's modulus.

The normal and shear strain-displacement relations are

$$
\begin{aligned}
\epsilon_{r r} & =\frac{\partial u_{r}}{\partial r}, \\
\epsilon_{\theta \theta} & =\frac{1}{r} \frac{\partial u_{\theta}}{\partial \theta}+\frac{u_{r}}{r},
\end{aligned}
$$

$\epsilon_{z z}=\frac{\partial u_{z}}{\partial z}$

$\gamma_{\theta z}=\frac{\partial u_{\theta}}{\partial z}+\frac{1}{r} \frac{\partial u_{z}}{\partial \theta}$

$\gamma_{z r}=\frac{\partial u_{z}}{\partial r}+\frac{\partial u_{r}}{\partial z}$

$\gamma_{\theta z}=\frac{1}{r} \frac{\partial u_{r}}{\partial \theta}+\frac{\partial u_{\theta}}{\partial r}-\frac{u_{\theta}}{r}$.

The kinetic energy is

$$
T=\frac{\rho}{2} \int_{V}\left[\left(\frac{\partial u_{r}}{\partial t}\right)^{2}+\left(\frac{\partial u_{\theta}}{\partial t}\right)^{2}+\left(\frac{\partial u_{z}}{\partial t}\right)^{2}\right] d V,
$$

where $\rho$ is the mass density per unit volume.

For linear, small deformation vibration, the displacement components assume temporal simple harmonic functions in the forms

$$
\begin{aligned}
& u_{r}(\bar{r}, \bar{\theta}, \bar{z}, t)=U_{r}(\bar{r}, \bar{\theta}, \bar{z}) \sin \omega t, \\
& u_{\theta}(\bar{r}, \bar{\theta}, \bar{z}, t)=U_{\theta}(\bar{r}, \bar{\theta}, \bar{z}) \sin \omega t, \\
& u_{z}(\bar{r}, \bar{\theta}, \bar{z}, t)=U_{z}(\bar{r}, \bar{\theta}, \bar{z}) \sin \omega t,
\end{aligned}
$$

where $U_{r}, U_{\theta}, U_{z}$ are the displacement amplitude functions and $\omega$ is the angular frequency of vibration.

The maximum strain and kinetic energy integral expressions $U_{\max }$ and $T_{\max }$ can be derived easily by substituting Eqs. (6a)-(6c) into Eqs. (2) and (5) and determining the extremum with respect to time $t$.

\section{Elastic energy functional and eigenvalue equation}

The displacement amplitude functions for a vibrating thick cylindrical shell can be expressed by a set of threedimensional (3-D) $p$-Ritz functions. These functions are the products of 2-D $p$-Ritz functions $\phi_{r}(\bar{\theta}, \bar{z}), \phi_{\theta}(\bar{\theta}, \bar{z}), \phi_{z}(\bar{\theta}, \bar{z})$

Lim et al:: Open cylindrical shells 1437 
for midsurface deformation and 1-D $p$-Ritz functions $\psi_{r}(\bar{r})$, $\psi_{\theta}(\bar{r}), \psi_{z}(\bar{r})$ for thickness deformation. The displacement amplitude functions are

$$
\begin{aligned}
& U_{r}(\bar{r}, \bar{\theta}, \bar{z})=\sum_{i=1}^{m} \sum_{j=1}^{n} c_{r}^{i j} \phi_{r}^{i}(\bar{\theta}, \bar{z}) \psi_{r}^{j}(\bar{r}), \\
& U_{\theta}(\bar{r}, \bar{\theta}, \bar{z})=\sum_{i=1}^{m} \sum_{j=1}^{n} c_{\theta}^{i j} \phi_{\theta}^{i}(\bar{\theta}, \bar{z}) \psi_{\theta}^{j}(\bar{r}), \\
& U_{z}(\bar{r}, \bar{\theta}, \bar{z})=\sum_{i=1}^{m} \sum_{j=1}^{n} c_{z}^{i j} \phi_{z}^{i}(\bar{\theta}, \bar{z}) \psi_{z}^{j}(\bar{r}),
\end{aligned}
$$

in which $c_{r}^{i j}, c_{\theta}^{i j}, c_{z}^{i j}$ are unknown coefficients.

An energy functional is defined as the difference of the maximum strain and kinetic energy components

$$
\Pi=U_{\max }-T_{\max } .
$$

Numerical frequency solutions can be obtained by minimizing this energy functional with respect to the coefficients in accordance with the Ritz procedure

$$
\frac{\partial \Pi}{\partial c_{\alpha}^{i j}}=0, \quad \alpha=r, \quad \theta, \quad \text { and } z,
$$

which leads to the governing eigenvalue equation

$$
\left(\mathbf{K}-\Lambda^{2} \mathbf{M}\right)\{\mathbf{C}\}=\{\mathbf{0}\},
$$

where

$$
\begin{aligned}
& \Lambda=\frac{\lambda}{\theta_{0}}, \\
& \lambda=\omega b \sqrt{\frac{\rho}{E}}
\end{aligned}
$$

are the dimensionless frequency parameters in which $b$ $=R \theta_{0}$ is the circumferential arc length.

The stiffness and mass matrices are

$$
\begin{gathered}
\mathbf{K}=\left[\begin{array}{ccc}
\mathbf{k}_{r r} & \mathbf{k}_{r \theta} & \mathbf{k}_{r z} \\
& \mathbf{k}_{\theta \theta} & \mathbf{k}_{\theta z} \\
\operatorname{sym} & & \mathbf{k}_{z z}
\end{array}\right], \\
\mathbf{M}=\left[\begin{array}{lll}
\mathbf{m}_{r r} & {[0]} & {[0]} \\
& \mathbf{m}_{\theta \theta} & {[0]} \\
\operatorname{sym} & & \mathbf{m}_{z z}
\end{array}\right],
\end{gathered}
$$

and the vector or unknown coefficients is

$$
\mathbf{C}=\left\{\begin{array}{l}
\left\{\mathbf{c}_{r}\right\} \\
\left\{\mathbf{c}_{\theta}\right\} \\
\left\{\mathbf{c}_{z}\right\}
\end{array}\right\} .
$$

The elements in the stiffness submatrix are

$$
\begin{aligned}
k_{r r}^{i k j l}= & \frac{\Delta+2 G}{E}\left[I_{\phi_{r r}^{i k} \psi_{r r}^{j l}}^{0000,11(1)}+I_{\phi_{r r}^{i k} \psi_{r r}^{j l}}^{0000,00(-1)}\right] \\
& +\frac{\Delta}{E}\left[I_{\phi_{r r}^{i k} \psi_{r r}^{j l}}^{0000,10(0)}+I_{\phi_{r r}^{i k} \psi_{r r}^{j l}}^{0000,01(0)}\right] \\
& +\frac{G}{E}\left[\frac{R^{2}}{a^{2}} I_{\phi_{r r}^{i k} \psi_{r r}^{j l}}^{0101,00(1)}+\frac{1}{\theta_{0}^{2}} I_{\phi_{r r}^{i k} \psi_{r r}^{j l}}^{1010,00(-1)}\right], \\
k_{r \theta}^{i k j l}= & \frac{\Delta+2 G}{E \theta_{0}} I_{\phi_{r \theta}^{i k} \psi_{r \theta}^{0010,00(-1)}}^{00}+\frac{\Delta}{E \theta_{0}} I_{\phi_{r \theta}^{i k} \psi_{r \theta}^{j l}}^{0010,10(0)} \\
& +\frac{G}{E \theta_{0}}\left[I_{\phi_{r \theta}^{i k} \psi_{r \theta}^{j l}}^{1000,01(0)}-I_{\phi_{r \theta}^{i k} \psi_{r \theta}^{j l}}^{1000,00(-1)}\right],
\end{aligned}
$$

$$
k_{r z}^{i k j l}=\frac{\Delta R}{E a}\left[I_{\phi_{r z}^{i k} \psi_{r z}^{j l}}^{0001,00(0)}+I_{\phi_{r z}^{i k} \psi_{r z}^{j l}}^{0001,10(1)}\right]+\frac{G R}{E a} I_{\phi_{r z}^{i k} \psi_{r z}^{j l}}^{0100,01(1)},
$$

$$
\begin{aligned}
k_{\theta \theta}^{i k j l}= & \frac{\Delta+2 G}{E \theta_{0}} I_{\phi_{\theta \theta}^{i k} \psi_{\theta \theta}^{j l}}^{1010,00(-1)}+\frac{G}{E}\left[\frac{R^{2}}{a^{2}} I_{\phi_{\theta \theta}^{i k} \psi_{\theta \theta}^{0101,00(1)}}^{j l}\right. \\
& \left.+I_{\phi_{\theta \theta}^{i k} \psi_{\theta \theta}^{000,11(1)}}^{j l(1)}+I_{\phi_{\theta \theta}^{i k} \psi_{\theta \theta}^{j 000,00(-1)}}^{000} I_{\phi_{\theta \theta}^{i k} \psi_{\theta \theta}^{j l l}}^{0000,10(0)}-I_{\phi_{\theta \theta}^{i k} \psi_{\theta \theta}^{j l}}^{0000,01(0)}\right],
\end{aligned}
$$

$$
\begin{aligned}
k_{\theta z}^{i k j l}= & \frac{R}{a \theta_{0}}\left[\frac{\Delta}{E} I_{\phi_{\theta z}^{i k} \psi_{\theta z}^{j l}}^{1001,00(0)}+\frac{G}{E} I_{\phi_{\theta z}^{i k} \psi_{\theta z}^{j l}}^{0110,00(0)}\right], \\
k_{z z}^{i k j l}= & \frac{(\Delta+2 G) R^{2}}{E a^{2}} I_{\phi_{z z}^{i k} \psi_{z z}^{j l}}^{0101,00(1)} \\
& +\frac{G}{E}\left[\frac{1}{\theta_{0}^{2}} I_{\phi_{z z}^{i k} \psi_{z z}^{j l}}^{1010,00(-1)}+I_{\phi_{z z}^{i k} \psi_{z z}^{j l}}^{0000,11(1)}\right],
\end{aligned}
$$

and the elements in the mass submatrix are

$$
\begin{aligned}
& m_{r r}^{i k j l}=I_{\phi_{r r}^{i k} \psi_{r r}^{j l}}^{0000,00(1)}, \\
& m_{\theta \theta}^{i k j l}=I_{\phi_{\theta \theta}^{i k} \psi_{\theta \theta}^{j l}}^{0000,00(1)}, \\
& m_{z z}^{i k j l}=I_{\phi_{z z}^{i k} \psi_{z z}^{j l}}^{0000,00(1)},
\end{aligned}
$$

in which

$$
\begin{aligned}
I_{\phi_{\alpha \beta}^{i k} \psi_{\alpha \beta}^{j l}}^{a b c d e, e f(g)}= & \iint_{\bar{A}} \frac{\partial^{a+b} \phi_{\alpha}^{i}(\bar{\theta}, \bar{z})}{\partial \bar{\theta}^{a} \partial \bar{z}^{b}} \frac{\partial^{c+d} \phi_{\beta}^{k}(\bar{\theta}, \bar{z})}{\partial \bar{\theta}^{c} \partial \bar{z}^{d}} d \bar{\theta} d \bar{z} \\
& \times \int_{\bar{t}} \frac{\partial^{e} \psi_{\alpha}^{j}(\bar{r})}{\partial \bar{r}^{e}} \frac{\partial^{f} \psi_{\beta}^{l}(\bar{r})}{\partial \bar{r}^{f}} \bar{r}^{g} d \bar{r},
\end{aligned}
$$

where $\alpha, \beta=r, \theta, z ; i, j, k, l=1,2, \ldots, m$, and $m$ is the total number of terms employed in the $p$-Ritz shape functions. The normalized midsurface area is denoted as $\bar{A}$ and the normalized thickness is $\bar{t}$. 
TABLE I. Convergence of $\lambda=\omega b \sqrt{\rho / E}$ for a thick cylindrical shell with $\nu=0.3, a / b=2, t / b=0.2$, and $\theta_{0}$ $=180^{\circ}$.

\begin{tabular}{cccccc}
\hline \hline & & \multicolumn{3}{c}{ Mode sequence number } \\
\cline { 3 - 5 } B.C. & $p_{\theta z} \times p_{r}$ & $\mathrm{~S}-1$ & $\mathrm{~S}-2$ & $\mathrm{~A}-1$ & A-2 \\
\hline CFFF & $8 \times 2$ & 0.093645 & 0.49279 & 0.15593 & 0.26904 \\
& $9 \times 2$ & 0.093542 & 0.49237 & 0.15580 & 0.26873 \\
& $10 \times 2$ & 0.093479 & 0.49207 & 0.15572 & 0.26851 \\
& $10 \times 3$ & 0.093425 & 0.49122 & 0.15509 & 0.26772 \\
& $10 \times 4$ & 0.093421 & 0.49119 & 0.15507 & 0.26769 \\
& & & & AS-1 & AA-1 \\
\hline \multirow{2}{*}{ CFCF } & $6 \times 2$ & 0.48287 & 0.46806 & 1.1378 & 1.0451 \\
& $8 \times 2$ & 0.48134 & 0.45980 & 1.0838 & 1.0196 \\
& $10 \times 2$ & 0.48068 & 0.45868 & 1.0805 & 1.0168 \\
& $10 \times 3$ & 0.47935 & 0.45416 & 1.0754 & 1.0040 \\
& $10 \times 4$ & 0.47927 & 0.45403 & 1.0752 & 1.0035 \\
& $6 \times 2$ & 2.6317 & 1.8671 & 2.5180 & 2.2833 \\
& $8 \times 2$ & 2.6314 & 1.8630 & 2.5165 & 2.2778 \\
& $10 \times 2$ & 2.6313 & 1.8620 & 2.5160 & 2.2768 \\
& $10 \times 3$ & 2.6034 & 1.8174 & 2.5144 & 2.2341 \\
& $10 \times 4$ & 2.5993 & 1.8113 & 2.5142 & 2.2285 \\
\hline \hline
\end{tabular}

\section{The 1-D and 2-D p-Ritz admissible functions}

The midsurface and thickness displacements denoted by $u_{r}(\bar{r}, \bar{\theta}, \bar{z}), u_{\theta}(\bar{r}, \bar{\theta}, \bar{z})$, and $u_{z}(\bar{r}, \bar{\theta}, \bar{z})$ are truncated finite series given in Eqs. (6a)-(6c). The midsurface deformation admissible functions are sets of geometrically compliant 2-D polynomials $\phi_{r}(\bar{\theta}, \bar{z}), \phi_{\theta}(\bar{\theta}, \bar{z})$, and $\phi_{z}(\bar{\theta}, \bar{z})$ derived such that the geometric boundary conditions are satisfied at the outset. They are composed of the product of a series of simple two-dimensional polynomials (degree of polynomial $\left.p_{\theta z}\right)$ and boundary-compliant basic functions $\phi_{r}^{b}(\bar{\theta}, \bar{z})$, $\phi_{\theta}^{b}(\bar{\theta}, \bar{z})$, and $\phi_{z}^{b}(\bar{\theta}, \bar{z})$. The latter are geometric expressions of the cylindrical shell boundary raised to an appropriate basic power in accordance with various boundary constraints. These 2-D $p$-Ritz admissible functions have been developed and formulated ${ }^{4-6}$ for shallow shell studies using a higher-order shell theory. Similarly, the 1-D thickness admissible functions $\psi_{r}(r), \psi_{\theta}(r)$, and $\psi_{z}(r)$ are the products of sets of 1-D polynomials (degree of polynomial $p_{r}$ ) and appropriate basic functions $\psi_{r}^{b}(r), \psi_{\theta}^{b}(r)$, and $\psi_{z}^{b}(r)$.

Classification of vibration modes is possible by grouping terms with odd and even powers in $\phi_{r}(\bar{\theta}, \bar{z}), \phi_{\theta}(\bar{\theta}, \bar{z})$, and $\phi_{z}(\bar{\theta}, \bar{z}) .{ }^{4,5}$ This tremendously reduces the number of terms in each series and thus the determinant size of the eigenvalue equation is considerably smaller. Huge computational effort can be saved as discussed in detail in the next section.

\section{RESULTS AND DISCUSSION}

\section{A. Convergence and comparison of eigenvalues}

The convergence characteristics of the dimensionless frequency parameter $\lambda$ are presented in Table I for CFFF (cantilevered), CFCF, and CCCC shells. In these cases, C denotes a clamped edge and $\mathrm{F}$ denotes a free edge with sequence from $\bar{z}=-0.5$ going anticlockwise (see Fig. 1). The vibration modes are classified into various symmetry classes. A CFFF shell has two symmetry classes while CFCF and
CCCC shells have four symmetry classes. The degrees of 2-D and 1-D polynomial, $p_{\theta z}$ and $p_{r}$, are related to the number of terms in each series by $m_{\theta z}=\left(p_{\theta z}+1\right)\left(p_{\theta z}+2\right) / 2$ and $m_{r}=p_{r}+1$. Classification of modes has a significant effect on the efficiency of algorithm as the determinant size of the eigenvalue problem can be greatly reduced and tremendous numerical computation can be saved while maintaining the same level of numerical accuracy.

It can be observed in Table I that good convergence of $\lambda$ has been achieved for admissible functions with $p_{\theta z} \times p_{r}$ $=10 \times 4$. These degrees of polynomial have been adopted for all subsequent calculation unless stated otherwise. All the eigenvalues converge downwards as expected because the Ritz method overestimates stiffness and vibration frequency and underestimates displacement. The determinant size is $792 \times 792$ without mode classification. With mode classification, it is only $432 \times 432$ for the symmetric class (S) and $360 \times 360$ for the antisymmetric class (A) for CFFF shell. For CFCF and CCCC shells, the determinant sizes are $252 \times 252$ for the SS class and $180 \times 180$ for the SA, AS, and AA classes. Details of vibration mode classification have been addressed in Lim et al. ${ }^{14,15}$

A comparison of frequency parameters with finiteelement solutions (FEM) is presented in Table II. The FEM solutions are obtained using LUSAS, a commercial finite element package, with two different elements. The QTS8 elements, ${ }^{22}$ are eight-node thick shell elements for the analysis of arbitrarily curved shell geometries. These elements take account of membrane, shear, and flexural deformations. The HX8M elements ${ }^{22}$ are eight-node three-dimensional isoparametric solid elements where the variation of stresses within an element is regarded as linear.

As observed in Table II, the $p$-Ritz solutions agree well with the FEM solutions and agreement is excellent with the solutions using HX8M elements. This is expected as the HX8M elements are 3-D elements which consider transverse normal stress similar to the present analysis. Convergence of 
TABLE II. Comparison of $\lambda=\omega b \sqrt{\rho / E}$ for a thick cylindrical shell with $\nu=0.3, t / b=0.2$, and $\theta_{0}=180^{\circ}$.

\begin{tabular}{|c|c|c|c|c|c|c|}
\hline \multirow[b]{2}{*}{ B.C. } & \multirow{2}{*}{$\frac{a}{b}$} & \multirow[b]{2}{*}{ Sources } & \multicolumn{4}{|c|}{ Mode sequence number } \\
\hline & & & S-1 & S-2 & A-1 & A-2 \\
\hline \multirow[t]{7}{*}{ CFFF } & \multirow[t]{3}{*}{1} & FEM $^{\mathrm{a}}$ & 0.34079 & 1.1483 & 0.40619 & 0.69750 \\
\hline & & FEM $^{\mathrm{b}}$ & 0.34295 & 1.1476 & 0.40706 & 0.70120 \\
\hline & & $3-\mathrm{D}$ & 0.34299 & 1.1347 & 0.40676 & 0.70092 \\
\hline & \multirow[t]{4}{*}{2} & FEM $^{\mathrm{a}}$ & 0.093042 & 0.48893 & 0.15497 & 0.26706 \\
\hline & & FEM $^{\mathrm{b}}$ & 0.093433 & 0.49583 & 0.15507 & 0.26792 \\
\hline & & 3-D & 0.093425 & 0.49122 & 0.15509 & 0.26772 \\
\hline & & & SS-1 & SA-1 & AS-1 & AA-1 \\
\hline \multirow[t]{6}{*}{$\mathrm{CFCF}$} & \multirow[t]{3}{*}{1} & FEM $^{\mathrm{a}}$ & 1.3101 & 1.1981 & 2.6625 & 2.5794 \\
\hline & & $\mathrm{FEM}^{\mathrm{b}}$ & 1.3386 & 1.2269 & 2.7589 & 2.6855 \\
\hline & & 3-D & 1.3235 & 1.2141 & 2.7000 & 2.6257 \\
\hline & \multirow[t]{3}{*}{2} & FEM $^{\mathrm{a}}$ & 0.47587 & 0.45196 & 1.0668 & 0.99628 \\
\hline & & FEM $^{\mathrm{b}}$ & 0.48591 & 0.45796 & 1.1029 & 1.0281 \\
\hline & & 3-D & 0.47935 & 0.45416 & 1.0754 & 1.0040 \\
\hline \multirow[t]{6}{*}{$\mathrm{CCCC}$} & \multirow[t]{3}{*}{1} & FEM $^{\mathrm{a}}$ & 2.8071 & 2.2988 & 3.6263 & 3.5733 \\
\hline & & $\mathrm{FEM}^{\mathrm{b}}$ & 2.9055 & 2.3672 & 3.6855 & 3.6826 \\
\hline & & 3-D & 2.8696 & 2.3383 & 3.6567 & 3.6346 \\
\hline & \multirow[t]{3}{*}{2} & FEM $^{\mathrm{a}}$ & 2.5140 & 1.7504 & 2.5110 & 2.1692 \\
\hline & & $\mathrm{FEM}^{\mathrm{b}}$ & 2.6422 & 1.8477 & 2.5243 & 2.2680 \\
\hline & & 3-D & 2.6034 & 1.8174 & 2.5144 & 2.2341 \\
\hline
\end{tabular}

${ }^{\mathrm{a}}$ LUSAS solutions with $30 \times 30$ QTS8 thick shell elements (Ref. 22).

${ }^{b}$ LUSAS solutions with $15 \times 15 \times 6$ HX8M 3-D isoparametric solid elements (Ref. 22).

the FEM solutions for $30 \times 30$ QTS8 elements and $15 \times 15 \times 6$ HX8M elements used in the computation have been checked. It is emphasized here that it takes $1.5-4 \mathrm{~h}$ to obtain a converged FEM solution using LUSAS while it takes less than a minute to obtain equally accurate solutions using the $p$-Ritz approach with mode symmetry classification. Noting that some of the finite-element solutions are higher than the 3-D Ritz solutions and keeping in mind that solutions from the Ritz approach are always upper-bounded, the 3-D Ritz solutions presented in Table II not only require considerably less time than the finite-element method, but are also more accurate.

\section{B. Vibration frequency and mode shapes}

A set of new results for the free vibration of CFFF cylindrical shells with aspect ratio $a / b$ varying from 1 to 2 , where $a$ and $b$ are the length and arc of the open shell, is presented in Figs. 2 and 3. The corresponding results for CFCF and CCCC cylindrical shells are presented in Figs. 4 and 5 , and 6 and 7 . The subtended angle $\theta_{0}$ ranges from $10^{\circ}$ to $180^{\circ}$. Because the aspect ratio is constant, the open shell radius varies while $\theta_{0}$ is changed, keeping the thickness $t$ constant. The idea or initiative of the authors is to investigate the effects of subtended angle while the deepness of an open shell is changed by bending a given plate with fixed length $a$ and width $b$.

In the figures, most of the frequencies have been computed using $p_{\theta z} \times p_{r}=10 \times 4$ while lower polynomial degrees have been employed for small $\theta_{0}$ as indicated in the figures because the matrix becomes ill conditioned. The fundamental mode for a CFFF shell is the S-1 mode while for CFCF and CCCC shells the fundamental mode switches from the SS- 1 mode to the SA-1 mode. The threshold of $\theta_{0}$ at which switching of fundamental mode occurs varies depending on $a / b$ and boundary conditions as indicated by the intersections of the SS-1 and SA-1 curves in Figs. 4-7, which are, respectively, $120^{\circ}, 160^{\circ}, 130^{\circ}$, and $120^{\circ}$, approximately. It

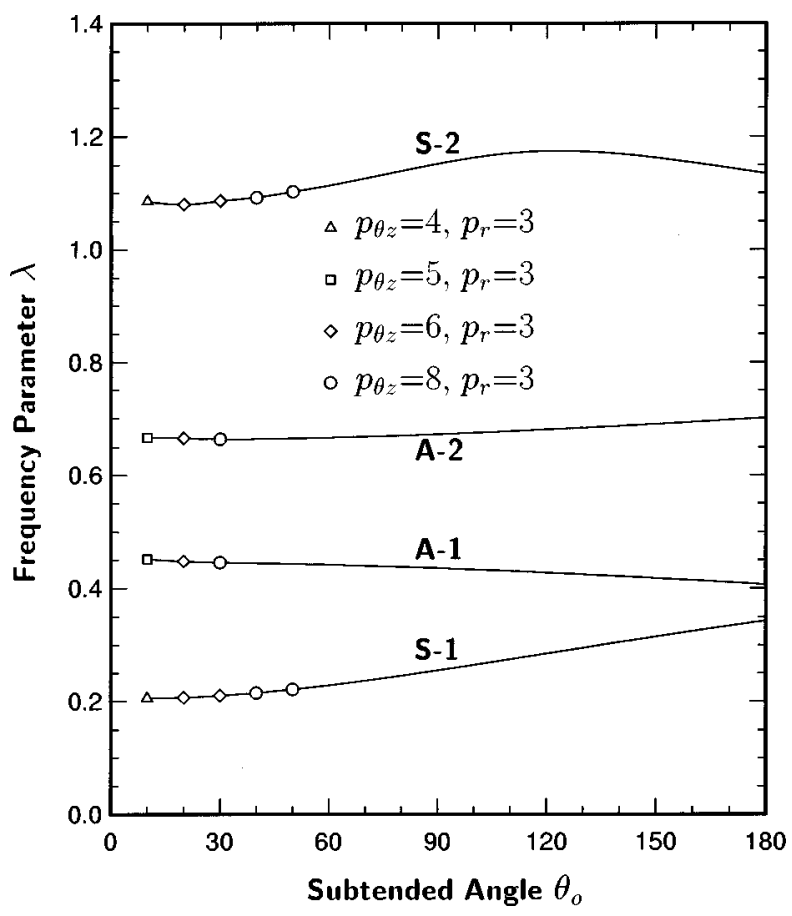

FIG. 2. Effect of subtended angle (degree) on frequency for a thick cylindrical shell (CFFF) with $\nu=0.3, t / b=0.2$, and $a / b=1$. 


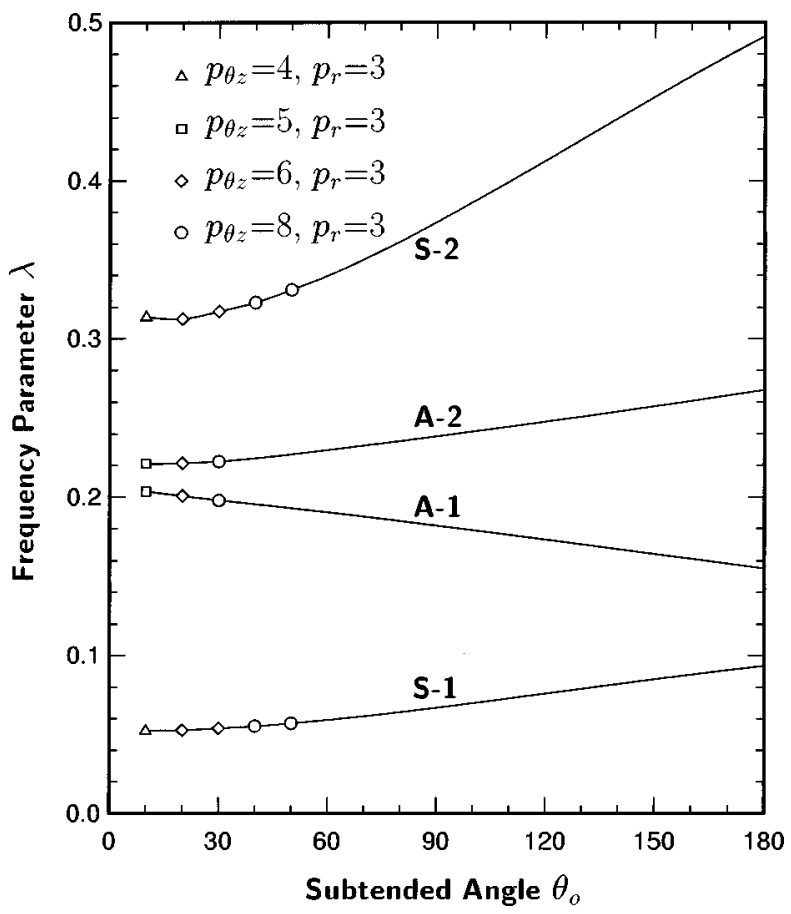

FIG. 3. Effect of subtended angle (degree) on frequency for a thick cylindrical shell (CFFF) with $\nu=0.3, t / b=0.2$, and $a / b=2$.

should be emphasized that the curves do not actually cross. If these curves were to cross, then, for some subtended angles, two different vibration modes would exist at the same frequency, which is indeed a violation of the uniqueness of a vibration mode. In this respect, computations have been conducted at very small intervals of $\theta_{0}$ and it is realized that the frequency curves belonging to different modes approach each other but they never coalesce. In fact, after the "coalescence," the frequency curves interchange their corre-

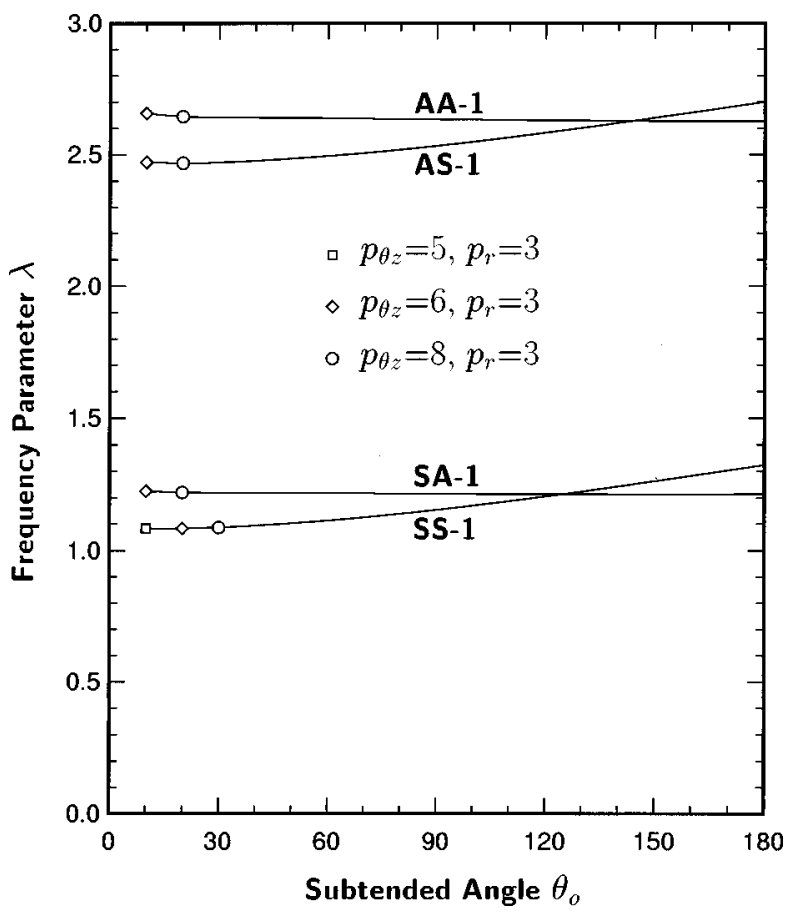

FIG. 4. Effect of subtended angle (degree) on frequency for a thick cylindrical shell (CFCF) with $\nu=0.3, t / b=0.2$, and $a / b=1$.

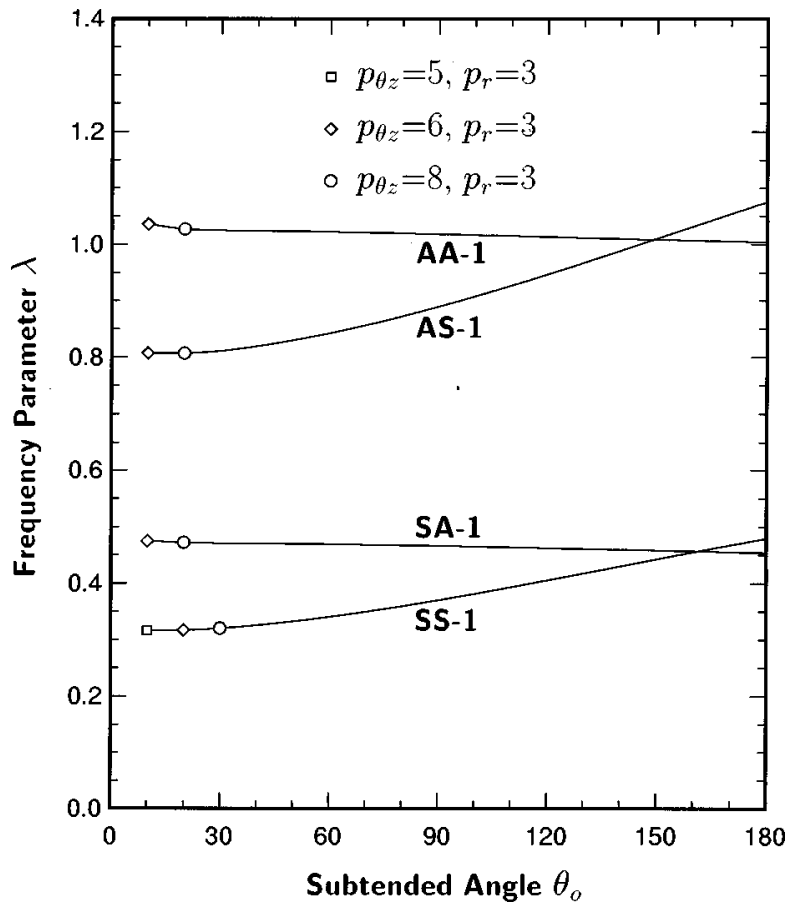

FIG. 5. Effect of subtended angle (degree) on frequency for a thick cylindrical shell (CFCF) with $\nu=0.3, t / b=0.2$, and $a / b=2$.

sponding types. This almost coalescence is not novel and it occurs in many problems whether in structural dynamics, acoustics, or fluid mechanics.

For a CFFF shell with $a / b=1$ and 2, the S-1 and A-2 frequencies increase with increasing $\theta_{0}$ while the A-1 frequency decreases. The S-2 frequency demonstrates irregular tendency with respect to $\theta_{0}$. For CFCF and CCCC shells, the SS-1 and AS-1 frequencies increase while the SA-1 and AA-1 frequencies decrease for shells with larger $\theta_{0}$. The

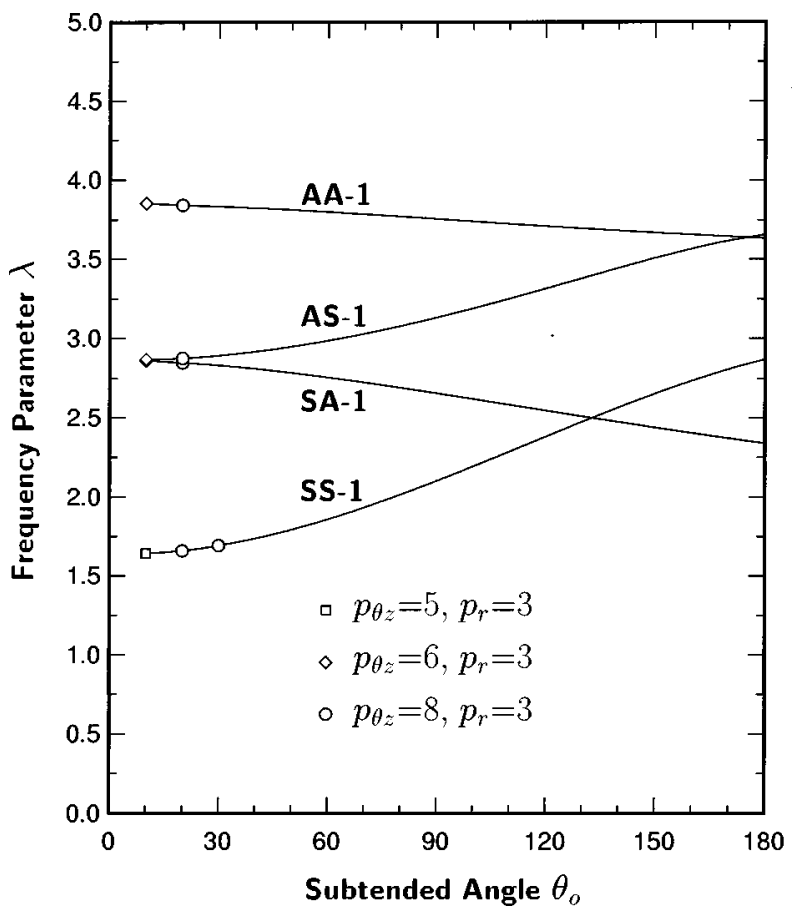

FIG. 6. Effect of subtended angle (degree) on frequency for a thick cylindrical shell (CCCC) with $\nu=0.3, t / b=0.2$, and $a / b=1$.

Lim et al:: Open cylindrical shells 


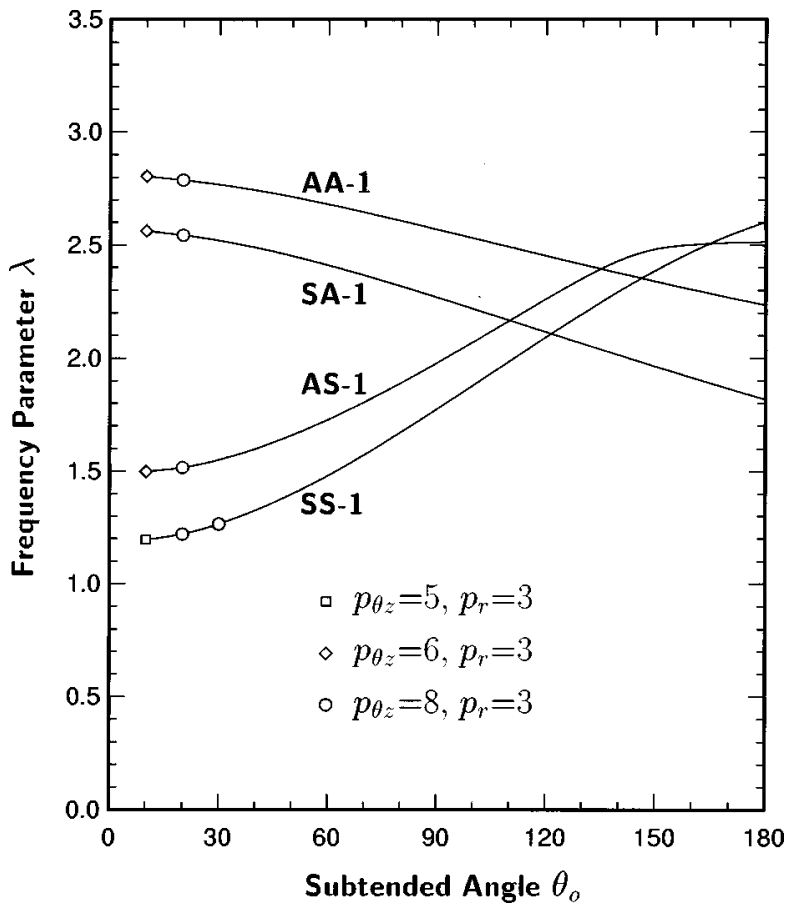

FIG. 7. Effect of subtended angle (degree) on frequency for a thick cylindrical shell (CCCC) with $\nu=0.3, t / b=0.2$, and $a / b=2$.

increasing rate of AS-1 frequency for a CCCC shell with $a / b=2$ (Fig. 7) decreases rapidly as $\theta_{0}$ approaches $150^{\circ}$ and onwards. In all these shell configurations, the frequency is smaller for longer shells (higher $a / b$ ) but it is larger for shells with stronger boundary constraints (from CFFF, CFCF to $\mathrm{CCCC}$ ).

New vibration mode shapes for the CFFF, CFCF, and CCCC shells are depicted in three-dimensional displacement meshes in Fig. 8. Various vibration modes can be observed from these figures, for instance, the S-1 and S-2 modes are the first and second flapwise bending modes. The A-1 and A-2 modes look alike but they are in fact the first twisting mode and the first in-surface mode in the circumferential direction, respectively. The free end of the A-1 mode is twisted while the free end of the A-2 mode is displaced in a circumferential direction. Because the cylinders are thick, not all lower frequency modes have the normal component, $U_{r}$, as the predominant component. For instance, the A-2 mode is circumferential dominant. The dependence of vibratory characteristics on boundary conditions are illustrated in these figures. The frequencies of a CFFF shell are lower than the CFCF and CCCC shells while the frequencies of a CCCC shell are the highest.

\section{CONCLUSIONS}

A new analysis method using a three-dimensional elasticity approach for free vibration of thick, open cylindrical shells has been developed. The spatial integrals for strain, with transverse normal stress, and kinetic energy components have been formulated. An energy functional has been defined and its extremum determined to arrive at a governing eigenvalue equation. The two-dimensional $p$-Ritz admissible functions previously used in thick shallow shell studies have

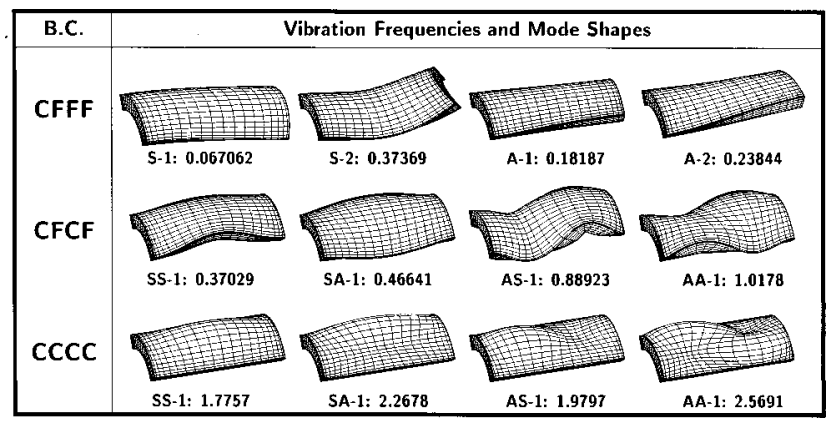

FIG. 8. Vibration frequencies $\lambda$ and mode shapes for a thick cylindrical shell with $\nu=0.3, t / b=0.2, a / b=2$, and $\theta_{0}=90^{\circ}$.

been generalized to three-dimensional functions by associating the two-dimensional functions with a one-dimensional $p$-Ritz admissible function.

Convergence of vibration frequencies has been examined and excellent comparison with finite-element solutions has been recorded. Classification of vibration modes by grouping terms in the $p$-Ritz functions tremendously reduces the matrix determinant size and thus much computation effort can been saved while maintaining the same level of accuracy. The algorithm developed here requires less than a minute to obtain accurate solutions which take hours for a finite-element package to compute. Most of the frequencies show consistent tendency with respect to increasing subtended angle. The frequency decreases for longer shells and shells with weaker boundary constraints. New threedimensional mode shapes have been presented.

\section{ACKNOWLEDGMENT}

The authors are grateful to anonymous reviewers for their valuable comments which helped improve the quality of this paper.

${ }^{1}$ E. Reissner, "The effect of transverse shear deformation on the bending of elastic plates," Trans. ASME, J. Appl. Mech. 12, A69-A77 (1945).

${ }^{2}$ R. D. Mindlin, "Influence of rotatory inertia and shear on flexural motions of isotropic, elastic plates," Trans. ASME, J. Appl. Mech. 18, 31-38 (1951).

${ }^{3}$ C. W. Lim and K. M. Liew, "Vibration of moderately thick cylindrical shallow shells," J. Acoust. Soc. Am. 100(6), 3665-3673 (1996).

${ }^{4}$ J. N. Reddy and C. F. Liu, "A higher-order shear deformation theory of laminated elastic shells," Int. J. Eng. Sci. 23, 319-330 (1985).

${ }^{5}$ C. W. Lim and K. M. Liew, "A higher order theory for vibration of shear deformable cylindrical shallow shells,", Int. J. Mech. Sci. 37(3), 277-295 (1995).

${ }^{6} \mathrm{~K}$. M. Liew and C. W. Lim, "A higher-order theory for vibration of doubly-curved shallow shells," Trans. ASME, J. Appl. Mech. 63(3), 587593 (1996).

${ }^{7}$ J. R. Hutchinson, "Axisymmetric vibration of a free finite-length rod," J. Acoust. Soc. Am. 51, 233-240 (1971).

${ }^{8}$ J. R. Hutchinson, "Transverse vibration of beams: Exact versus approximate solutions," Trans. ASME, J. Appl. Mech. 48, 923-928 (1981).

${ }^{9}$ A. W. Leissa and J. So, "Comparisons of vibration frequencies for rods and beams from one-dimensional and three-dimensional analyses," J. Acoust. Soc. Am. 98, 2122-2135 (1995).

${ }^{10}$ J. A. Fromme and A. W. Leissa, "Free vibration of the rectangular parallelepiped," J. Acoust. Soc. Am. 48, 290-298 (1970).

${ }^{11}$ S. Srinivas, C. V. Rao, and A. K. Rao, "An exact analysis for vibration of simply supported homogeneous and laminated thick rectangular plates," J. Sound Vib. 12, 187-199 (1970).

${ }^{12}$ J. R. Hutchinson and S. D. Zillmer, "Vibration of a free rectangular parallelepiped," Trans. ASME, J. Appl. Mech. 50, 123-130 (1983). 
${ }^{13}$ A. W. Leissa and Z. D. Zhang, "On the three-dimensional vibrations of the cantilevered rectangular parallelepiped,' J. Acoust. Soc. Am. 73, 2013-2021 (1983).

${ }^{14}$ C. W. Lim, K. H. Liew, and S. Kitipornchai, "Numerical aspects for free vibration of thick plates. Part I: Formulation and verification,' Comput. Methods Appl. Mech. Eng. 156(1-4), 15-29 (1998).

${ }^{15}$ C. W. Lim, S. Kitipornchai, and K. M. Liew, "Numerical aspects for free vibration of thick plates. Part II: Numerical efficiency and vibration frequencies,' Comput. Methods Appl. Mech. Eng. 156(1-4), 31-44 (1998).

${ }^{16}$ K. M. Liew, K. C. Hung, and M. K. Lim, "Free vibration studies on stress-free three-dimensional elastic solids," Trans. ASME, J. Appl. Mech. 62(1), 159-165 (1995).

${ }^{17}$ J. R. Hutchinson, "Vibrations of solid cylinders," Trans. ASME, J. Appl. Mech. 47, 901-907 (1980).
${ }^{18}$ K. M. Liew, K. C. Hung, and M. K. Lim, "Vibration of stress-free hollow cylinders of arbitrary cross section,', Trans. ASME, J. Appl. Mech. 62(3), 718-724 (1995).

${ }^{19}$ A. W. Leissa and J. So, "Accurate vibration frequencies of circular cylinders from three-dimensional analysis," J. Acoust. Soc. Am. 98(4), 2136-2141 (1995).

${ }^{20}$ J. So and A. W. Leissa, "Free vibrations of thick hollow circular cylinders from three-dimensional analysis," Trans. ASME, J. Vib. Acoust. 119, 89-95 (1997).

${ }^{21}$ A. W. Leissa and J. So, "Three-dimensional vibrations of truncated hollow cones,"' J. Vib. Control 1, 145-158 (1995).

${ }^{22}$ LUSAS Manual, Version II, Finite Element Library (FEA Ltd., Surrey, United Kingdom), pp. 134-138, 183-190. 